\title{
Additive Schwarz Methods for Elliptic Mortar Finite Element Problems
}

\author{
Maksymilian Dryja \\ Warsaw University, Department of Mathematics, \\ Banacha 2, 02-097 Warsaw, Poland. E-mail: dryja@mimuw.edu.pl
}

\begin{abstract}
Domain decomposition methods are designed and analyzed as additive Schwarz methods for the linear systems arising from the discretization of elliptic problems. The discretization is obtained by a mortar element method with a finite element approximation on a nonmatching triangulation. The additive Schwarz methods use inexact solvers and they can also be applied to elliptic problems with discontinuous coefficients.
\end{abstract}

\section{Keywords}

Domain decomposition, elliptic mortar finite element problems, preconditioned conjugate gradients, additive Schwarz methods, iterative substructuring.

\section{INTRODUCTION}

In this paper, we discuss a parallel algorithm for solving systems arising from the discretization of elliptic problems by the mortar element method; see Bernardi, Maday, and Patera (1994), Bernardi and Maday (1995). Our algorithm is a domain decomposition method and it is described as an additive Schwarz method (ASM); see Dryja and Widlund (1987) and Dryja, Smith, and Widlund (1994).

The mortar element method is applied to elliptic self-adjoint second order equations with Dirichlet boundary conditions in 2 -D. To simplify the presentation only certain model problems are discussed. The mortar element method is considered in the geometrically conforming case as well as the nonconforming case. The first case corresponds to a partition of the region into triangular or rectangular subregions that form a coarse finite element triangulation, while in the second case a vertex of a subregion can fall in the interior of an edge of one of its neighbors. In the first case a model problem with discontinuous coefficients is discussed while in the second only problems with regular coefficients are considered. A finite element method based on piecewise linear continuous functions is introduced for each subregion without insisting on meshes matching across subregion interfaces. 
The resulting linear systems are solved by an iterative substructuring method described as an additive Schwarz method using the general framework given in Dryja, Smith, and Widlund (1994). First, the unknowns corresponding to interior nodal points in each substructure are eliminated. The reduced problem is defined on the interfaces of the substructures and it is solved by an ASM with a special coarse space and inexact solvers. It is shown that the method is almost optimal from the point of view of parallel computations.

There are several papers devoted to solving systems arising from the mortar element method by domain decomposition methods; see Achdou, Maday and Widlund (1995), Widlund (1995) and the literature therein. Some of the results of this paper, which have been obtained independently, are close those of Achdou, Maday, and Widlund (1995). The main results of our paper is the development of simple inexact solvers for both the geometrically conforming and nonconforming case, and the analysis of the method for problems with discontinuous coefficients in the geometrically conforming case. These results are, to our knowledge, new.

The outline of the paper is as follows. In Section 2, the discrete problem is formulated and its properties are discussed including the condition number of stiffness matrix arising from the discrete problem. This is done for the geometrically conforming case. In Section 3 , an ASM for the discrete problem is designed. The analysis of it is carried out in Section 5. To do so, several technical tools are needed. They are formulated and proved in Section 4 .

The geometrically nonconforming case is discussed in Sections 6 and 7 where a description and analysis of the method can be found. For the analysis, the stability of a special projection is proved for functions which do not vanish at the vertices of the substructures. This very important and new result is crucial for the analysis.

Some of the results of this paper have been obtained in joint work with Olof Widlund.

\section{DIFFERENTIAL AND DISCRETE PROBLEMS}

To simplify our presentation, we consider only a model problem in 2-D, a special secondorder problem with discontinuous, piecewise constant coefficients:

Find $u \in H_{0}^{1}(\Omega)$ such that

$a(u, v)=f(v), \quad v \in H_{0}^{1}(\Omega)$,

where

$a(u, v)=\int_{\Omega} \rho(x) \nabla u \cdot \nabla v d x, \quad f(v)=\int_{\Omega} f v d x$.

The region $\Omega$ is polygonal and a union of nonoverlapping subregions $\Omega_{i} i=1, \ldots, N$, also called substructures.

We first consider the case when $\Omega$ is a union of triangles or rectangles $\Omega_{i}, i=1, \ldots, N$, which form a coarse triangulation. We assume that jumps of $\rho(x)>0$ occur only at substructure interfaces and, for simplicity, that the coefficient takes on a constant value $\rho(x)=\rho_{i}, x \in \Omega_{i}$, in each substructure. We can generalize our results to the case when the relative variation of $\rho(x)$ over each subregion is modest.

To define a discrete problem by the mortar element method, we introduce two partitions of $\Omega$, a coarse and a fine. As mentioned above, the coarse partition is formed by 
the substructures $\Omega_{i}$. To simplify our presentation, we assume that it is quasi-uniform with a parameter $H$, see Ciarlet (1978). To obtain the fine partition of $\Omega$, we introduce a triangulation of each $\Omega_{i}$ with triangular elements $e_{i}^{(k)}$. The resulting triangulation is nonmatching; the triangulation of the different $\Omega_{i}$ generally do not match on the interfaces between subregions. We assume that the triangulation of each $\Omega_{i}$ is quasi-uniform with a parameter $h_{i}$. Let $X_{i}^{h_{i}}\left(\Omega_{i}\right)$ be the finite element space of piecewise linear continuous functions defined on the triangulation of $\Omega_{i}$ vanishing on $\partial \Omega_{i} \cap \partial \Omega$. Let

$X^{h}(\Omega)=X_{1}^{h_{1}}\left(\Omega_{1}\right) \times \ldots \times X_{N}^{h_{N}}\left(\Omega_{N}\right)$

be a Hilbert space with the inner product

$a\left(u_{h}, v_{h}\right)=\sum_{i=1}^{N} \rho_{i} \int_{\Omega_{i}} \nabla u_{i, h} \nabla v_{i, h} d x$

where $u_{h}=\left\{u_{i, h}\right\}_{i=1}^{N}, u_{i, h} \in X_{i}^{h_{i}}\left(\Omega_{i}\right)$.

To describe the discrete problem fully, we introduce some auxiliary notations and finite element spaces. Let $\bar{\Gamma}_{i j}=\bar{\Omega}_{i} \cap \bar{\Omega}_{j}$ and let $W_{i j}^{h_{i}}\left(\Gamma_{i j}\right)$ and $W_{j i}^{h_{j}}\left(\Gamma_{i j}\right)$ be the restrictions of $X_{i}^{h_{i}}\left(\Omega_{i}\right)$ and $X_{j}^{h_{j}}\left(\Omega_{j}\right)$ to $\Gamma_{i j}$. Note that the functions of these spaces are discontinuous across $\Gamma_{i j}$, a side common to $\Omega_{i}$ and $\Omega_{j}$. To define weak continuity across the interface, we introduce mortar (master) and nonmortar (slave) sides $\Gamma_{i j}$. Let the master side be $\Gamma_{i j}$ a side of the substructure $\Omega_{i}$ where the coefficient $\rho(x)$ is larger i.e. $\rho_{i} \geq \rho_{j}$. Thus $\Gamma_{i j}$ also forms a slave side of $\Omega_{j}$. We now introduce the master, slave, and test spaces. The spaces $W_{i j}^{h_{i}}\left(\Gamma_{i j}\right)$ and $W_{i j}^{h_{j}}\left(\Gamma_{i j}\right)$ are the master and slave ones. The test space, denoted by $M_{i j}^{h_{j}}\left(\Gamma_{i j}\right)$, is a subspace of $W_{i j}^{h_{j}}\left(\Gamma_{i j}\right)$ and its functions are constant on the elements $e_{j}^{(k)}$ at the ends of $\Gamma_{i j}$; the dimension of $M_{i j}^{h_{j}}\left(\Gamma_{i j}\right)$ is two less than that of $W_{i j}^{h_{j}}\left(\Gamma_{i j}\right)$. We are now in position to define a space $V^{h}(\Omega)$ used for the discretization of (1) by the mortar element method. $V^{h}$ is the space of $u_{h} \in X^{h}$ such that for $i=1, \ldots, N$ and each slave side $\Gamma_{i j}$ the following mortar condition

$\int_{\Gamma_{i j}}\left(u_{i, h}-u_{j, h}\right) \Psi d s=0, \quad \Psi \in M_{i j}^{h_{j}}\left(\Gamma_{i j}\right)$

is satisfied. Here $u_{i, h}$ and $u_{j, h}$ in the integral are the restrictions to $\Gamma_{i j}$, the common side of $\Omega_{i}$ and $\Omega_{j}$, of the the finite element functions. This imposes a weak continuity constraint of $u_{i, h}$ and $u_{j, h}$ on $\Gamma_{i j}$. It also means that the $L_{2}$-projections of $u_{i, h}$ and $u_{j, h}$, restricted to $\Gamma_{i j}$, onto $M_{i j}^{h_{j}}\left(\Gamma_{i j}\right)$ are equal to each other.

The discrete problem is of the form:

Find $u_{h} \in V^{h}$ such that

$a\left(u_{h}, v_{h}\right)=f\left(v_{h}\right), \quad v_{h} \in V^{h}$.

This problem has a unique solution and

$\sum_{i=1}^{N}\left\|u-u_{i, h}\right\|_{H^{1}\left(\Omega_{i}\right)}^{2} \leq C \sum_{i=1}^{N} h_{i}^{2}|u|_{H^{2}\left(\Omega_{i}\right)}^{2}$,

provided that $u \in H_{0}^{1}(\Omega) \cap H^{2}\left(\Omega_{i}\right)$; see Bernardi, Maday and Patera (1994), Belgacem (1995), Bernardi and Maday (1995). 
Our goal is to design and analyze a parallel algorithm for solving the discrete problem. It is a domain decomposition method, more exactly, an additive Schwarz method which is almost optimal from the point of view of parallel computations; see Dryja, Smith, and Widlund (1994). The first question concerns the condition number of the stiffness matrix $A$ of the system resulting from (3). It has been established, independently by Bernardi and Maday (1995) and by Dryja and Widlund (1995) that it is of the same order as in for conforming finite element approximations, i.e.

$\operatorname{cond}(A) \leq C h^{-2}$.

Here $h$ is the smallest of the $h_{i}$.

\section{ADDITIVE SCHWARZ METHODS}

In this section, we describe an iterative substructuring method in terms of an additive Schwarz method (ASM). For that the general framework of ASM is used, see Dryja, Smith, and Widlund (1995).

In a first step of the method considered, all unknowns associated with interior nodal points of $\Omega_{i}$ are eliminated. For $i=1, \ldots, N u_{i, h} \in X_{i}^{h_{i}}\left(\Omega_{i}\right)$ is represented as

$u_{i, h}=P u_{i, h}+H u_{i, h}$,

where $P u_{i, h}$ is the $H_{0}^{1}\left(\Omega_{i}\right)$ - projection onto $X_{i}^{h_{i}}\left(\Omega_{i}\right)$ while $H u_{i, h}$ is the discrete harmonic function defined by

$$
\left(\nabla H u_{i, h}, \nabla v_{i, h},\right)_{L_{2}\left(\Omega_{i}\right)}=0, \quad v \in \stackrel{0}{X_{i}^{h_{i}}}\left(\Omega_{i}\right)
$$

with $H u_{i, h}=u_{i, h}$ on $\partial \Omega_{i}$. Here $\stackrel{0}{X}_{i}^{h_{i}}\left(\Omega_{i}\right)$ is the subspace of functions of $X_{i}^{h_{i}}\left(\Omega_{i}\right)$ which vanish on $\partial \Omega_{i}$. Using this approach, we reduce the problem (3) to the following: (Let $V_{1}^{h}(\Omega)$, a subspace of $V^{h}(\Omega)$, be a space of discrete harmonic functions in each $\Omega_{i}$.)

Find $u_{h} \in V_{1}^{h}(\Omega)$ such that

$s\left(u_{h}, v_{h}\right)=\tilde{f}\left(v_{h}\right), \quad v_{h} \in V_{1}^{h}(\Omega)$,

where

$s\left(v_{h}, w_{h}\right)=a\left(v_{h}, w_{h}\right), \quad u_{h}, v_{h} \in V_{1}^{h}(\Omega) \quad$ and $\quad \tilde{f}\left(v_{h}\right)=f\left(v_{h}\right)-a\left(P u_{h}, v_{h}\right)$.

The problem (8) has a unique solution and it can be proved that the condition number of the matrix $S$, resulting from (8), is of the order of $h^{-1}$ provided that the $h_{i}$ are of the order of $h$.

For solving (8), we design and analyze an ASM. For that we use the general framework, see Dryja, Smith, and Widlund (1994), which describes the method in terms of a decomposition of $V_{1}^{h}$ into subspaces, certain bilinear forms given on these subspaces, and the projections onto these subspaces in the sense of the bilinear forms. The decomposition of $V_{1}^{h}$ is taken as

$V_{1}^{h}=V_{10}+V_{11}$

where $V_{11}$ is a space of functions belonging to $V_{1}^{h}$ vanishing at the vertices of the substructures $\Omega_{i}$, i.e. the nodal points of the coarse triangulation. To define the space $V_{10}$, let 
us first discuss the case when functions of $V_{1}^{h}$ are continuous at the vertices of $\Omega_{i}$, which are assumed to be triangles. In this case $V_{10}=V^{H}$, is a conforming finite element space defined on the coarse triangulation. We now define $V_{10}$ without assuming continuity at the vertices. Let $x_{i j l}$ be a vertex of $\Omega_{i}$ and the end of $\Gamma_{i j}$ and $\Gamma_{i l}$, sides of $\Omega_{j}$ and $\Omega_{l}$ common with $\Omega_{i}$. We should denote that point by $x_{i j \ldots k}$ since it is common to the substructures $\Omega_{i}, \Omega_{j}, \ldots, \Omega_{k}$. For simplicity let us denote only by $x_{i j l}$. We associate with $x_{i j l}$ a basis function $\varphi_{i j l}$ defined as follows. Let $\Gamma_{i j}$, a side of $\Omega_{i}$ with one end as $x_{i j l}$, be the master one. We introduce $\varphi_{i j}^{(m)}$ as a linear function defined on $\Gamma_{i j}$ with the values one at $x_{i j l}$ and zero at the other end of $\Gamma_{i j}$. The function $\varphi_{i j}^{(m)}$ is extended to the slave side of $\Omega_{j}$ (common with $\Omega_{i}$ ) by using the mortar condition. That extension is denoted by $\varphi_{i j}^{(m s)}$ and defined by

$\int_{\Gamma_{i j}} \varphi_{i j}^{(m s)} \Psi d s=\int_{\Gamma_{i j}} \varphi_{i j}^{(m)} \Psi d s, \quad \Psi \in M_{i j}^{h_{j}}\left(\Gamma_{i j}\right)$,

with the additional condition that $\varphi_{i j}^{(m s)}(x)$ vanishes at the ends of $\Gamma_{i j}$. If $\Gamma_{i l}$ is a slave (nonmortar) side of $\Omega_{i}$, we associate with $x_{i j l}$ a function $\varphi_{i l}^{(s)}$ defined by

$\int_{\Gamma_{i l}} \varphi_{i l}^{(s)} \Psi d s=0, \quad \Psi \in M_{i l}^{h_{i}}\left(\Gamma_{i l}\right)$

with the condition that $\varphi_{i j}\left(x_{i j l}\right)=1$ and it vanishes at the other end of $\Gamma_{i j}$. This means that $\varphi_{i j}^{(s)}$ is the image of zero defined on the master side $\Gamma_{i l}$ belonging to $\Omega_{l}$. The function $\varphi_{i j l}(x)$ associated with the vertex $x_{i j l}$ is the discrete harmonic function in each substructure with the following boundary data: $\varphi_{i j l}(x)=0$ on all sides of the substructures except those for which $x_{i j l}$ is a common vertex. On the sides with the common vertex $\varphi_{i j l}$ is: for a master side $\Gamma_{i j}$ it is equal to $\varphi_{i j}^{(m)}$ and for a slave side of $\Omega_{i}$ common with $\Omega_{l}$ (which is the master for $\left.\Omega_{l}\right)$ it is equal to $\varphi_{i l}^{(s)}+\varphi_{i l}^{(m s)}$. Of course, $\varphi_{i j l} \in V_{1}^{h}$ and

$V_{10}=\operatorname{span}\left\{\varphi_{i j l}\right\}$.

We now introduce bilinear forms $b_{1 k}, k=0,1$, related to the inexact solvers over $V_{1 k} \times V_{1 k}$, which approximate $a(u, v)$. One possibility to define $b_{11}(u, v)$ is

$b_{11}\left(u_{h}, v_{h}\right)=\sum_{\Omega_{i}} \sum_{\Gamma_{i j} \subset \partial \Omega_{i}} \rho_{i}\left(K_{i j}^{1 / 2} \underline{u}_{i, h}^{(m)}, \underline{v}_{i, h}^{(m)}\right)_{R^{N_{i j}}}$.

Here the sum is taken over the master sides of $\Omega_{i}, u_{i, h}^{(m)}=u_{i, h}$ when $\Gamma_{i j}$ is the master side of $\Omega_{i} . \underline{u}_{i, h}^{(m)}$ is the vector representation of $u_{i, h}$ on $\Gamma_{i j}$ using the standard nodal basis functions associated with the interior nodal points of $\Gamma_{i j}$ corresponding to the triangulation on the master side; the number of nodes is denoted by $N_{i j}$. The matrix $K_{i j}$ is the matrix representation of $(\nabla u, \nabla v)_{L^{2}(0, l)}$, approximated by the conforming finite element space of piecewise linear continuous functions which vanish at the ends of $(0, l)$, defined on triangulation on $(0, l)$ with uniform step and $N_{i j}$ the number of interior nodal points. Note that the system with $K_{i j}^{1 / 2}$ can be solved using FFT, for details see, e.g. Dryja (1982).

The other possibility to define $b_{11}\left(u_{h}, v_{h}\right)$ is given by

$b_{11}\left(u_{h}, v_{h}\right)=\sum_{\Omega_{i}} \sum_{\Gamma_{i j} \subset \partial \Omega_{i}} b_{\Gamma_{i j}}\left(u_{i, h}^{(m)}, v_{i, h}^{(m)}\right)$, 
where the sum, as in (13), is taken over the master sides $\Gamma_{i j}$ of $\partial \Omega_{i}$ and

$b_{\Gamma_{i j}}\left(u_{i, h}^{(m)}, v_{i, h}^{(m)}\right)=\rho_{i}\left(\nabla u_{i, h}^{(m)}, \nabla v_{i, h}^{(m)}\right)_{L_{2}\left(\Omega_{i}\right)}$

Here $\Gamma_{i j}$ is the master side of $\Omega_{i}$ and $u_{i, h}^{(m)}$ is equal to $u_{i, h}$ on $\Gamma_{i j}$ at the interior points and zero on the remaining sides of $\partial \Omega_{i}$ and others. Note that the solving of a problem with the form $b_{11}$ reduces to solving of local conforming finite element problems defined only on individual substructures and that these problems are independent.

The form $b_{10}\left(u_{h}, v_{h}\right), u_{h}, v_{h} \in V_{10}$, is given by

$$
\begin{aligned}
b_{10}\left(u_{h}, u_{h}\right) & =\sum_{\Omega_{i}}\left\{\sum_{x \in \nu_{i}}\left(1+\log \frac{H}{h_{i}}\right) \rho_{i}\left(u_{i, h}(x)-\bar{u}_{i, h}\right)^{2}\right. \\
& \left.+\sum_{\Gamma_{i j} \subset \partial \Omega_{i}} \sum_{x \in \nu_{i j}}\left(1+\log \frac{H}{h_{j}}\right) \rho_{j}\left(u_{i, h}^{(m)}(x)-\bar{u}_{j, h}^{(s)}\right)^{2}\right\},
\end{aligned}
$$

where the sum is taken over all substructures $\Omega_{i}$ while in the second term it is taken over all the master sides $\Gamma_{i j}$ belonging to $\Omega_{i}$. Here $\nu_{i}$ is a set of vertices of $\Omega_{i}$ and $\nu_{i j}$ is the ends of $\Gamma_{i j} ; \bar{u}_{i, h}$ is the average of $u_{i, h}$ at vertices of $\Omega_{i}$, i.e.

$\bar{u}_{i, h}=\frac{1}{n_{i}} \cdot \sum_{x \in \nu_{i}} u_{i, h}(x)$

where $n_{i}$ is the number of vertices of $\Omega_{i} ; u_{i, h}^{(m)}=u_{i, h}$ when $\Gamma_{i j}$ is the master side of $\Omega_{i}$ and $u_{j, h}^{(s)}=u_{j, h}$ when $\Gamma_{i j}$ is a slave side of $\Omega_{j}$, common with $\Omega_{i}$.

We now are in position to define approximate projections $T_{1 k}: V_{1}^{h} \rightarrow V_{1 k}, k=0,1$, as

$b_{1, k}\left(T_{k} u, v\right)=a(u, v), \quad v \in V_{1 k}$

and we denote

$T_{1}=T_{10}+T_{11}$

where $T_{1}: V^{h} \rightarrow V^{h}$.

The original problem (8) can be replaced by

$T_{1} u_{h}=g_{1}$

where $g_{1}=g_{10}+g_{11}$ which are computed as the solutions of

$b_{1 k}\left(g_{1 k}, v\right)=f(v), \quad v \in V_{1 k}$.

The problems (20) and (8) have the same unique solution since $T_{1}$ is invertible; see Theorem 1 below.

To find $u_{h}$, the solution of (8), we solve the problem (20) iteratively since the operator 
$T_{1}$ is symmetric, positive definite and well conditioned, which follows from Theorem 1. We now formulate the main result of this section.

Theorem 1 For any $v_{h} \in V_{1}^{h}$

$c \delta^{-2} a\left(v_{h}, v_{h}\right) \leq\left(T_{1} v_{h}, v_{h}\right) \leq C a\left(v_{h}, v_{h}\right)$

where $c$ and $C$ are positive constants independent of $H, h_{i}$ and the jumps of $\rho(x), \delta=$ $\left(1+\log \frac{H}{h}\right)$ and $h=\inf _{i} h_{i}$.

A proof of Theorem 1 is given in Section 5. We now discuss an implementation of the method. For solving (20), we can use the conjugate gradient method since $T_{1}$ is symmetric, positive definite and its condition number is almost constant. To simplify our presentation, we use the Richardson method for solving (20):

$u_{h}^{n+1}=u_{h}^{n}-\tau_{o p t}\left(T_{1} u_{h}^{n}-g_{1}\right)$,

where $\tau_{o p t}$ is the relaxation parameter. To find $u_{h}^{n+1}$, we need to compute

$r_{1}=T_{1} u_{h}^{n}-g_{1}=T_{1}\left(u_{h}^{n}-u_{h}\right)=r_{10}+r_{11}$,

where $r_{1 k}=T_{1 k}\left(u_{h}^{n}-u_{h}\right), k=0,1$, are the solutions of

$b_{1 k}\left(r_{1 k}, v_{h}\right)=a\left(u_{h}^{n}, v_{h}\right)-\tilde{f}\left(v_{h}\right) \equiv F\left(v_{h}\right), \quad v_{h} \in V_{1 k}$.

These two problems are independent. To find $r_{10}$, we solve a global system of dimension equal to the number of values of $u_{h}$ at the vertices of the substructures.

The solution of $(23)$, for $k=1$, reduces to solving a set of local subproblems defined in one or two substructures. Let us give some details in the case when $b_{11}$ is given by (13). For a fixed $\Gamma_{i j}$, we solve the equation

$\left(K_{i j}^{1 / 2} \underline{r}_{i}^{(m)}, \underline{v}_{i}^{(m)}\right)=g\left(v_{h}\right), \quad v_{h} \in V_{11}$,

where $r_{i}^{(m)}=r_{11}$ on $\Gamma_{i j}$, using the scheme:

1. For the nodal points $x_{K} \in \Gamma_{i j}$, the master side of $\Omega_{i}$, take a basis function $\Phi_{K} \in V_{1}$ which is equal to the discrete harmonic function on $\bar{\Omega}_{i}$ with values one at $x_{K}$ and zero at the remaining nodal points of $\partial \Omega_{i}$. On the slave side $\Gamma_{i j}$ of $\Omega_{j}, \Phi_{K}$ is an extension using the mortar condition (2) with $\Phi_{K}(x)=0$ at the ends of $\Gamma_{i j}$. Then $\Phi_{K}(x)$ on $\Omega_{j}$ is the discrete harmonic extension with zero on $\partial \Omega_{j} \backslash \Gamma_{i j}$. On the remaining substructures $\Phi_{K}(x)=0$. The system so obtained is solved by FFT and it gives the values of $r_{i}^{(m)}(x)=r_{11}(x)$ at the nodal points of $\Gamma_{i j}$, the master side.

2. Find $r_{j}(x)$ at the nodal points of $\Gamma_{i j}$, the slave side of $\Omega_{j}$, solving the problem

$$
\int_{\Gamma_{i j}} r_{j}(x) \Psi d s=\int_{\Gamma_{i j}} r_{i}^{(m)}(x) \Psi d s, \quad \Psi \in M_{i j}^{h_{j}}\left(\Gamma_{i j}\right)
$$


with $r_{j}(x)=0$ at the ends of $\Gamma_{i j}$. For that, we use the standard nodal basis functions of $W_{i j}^{h_{j}}\left(\Gamma_{i j}\right)$ and $M_{i j}^{h_{j}}\left(\Gamma_{i j}\right)$. That gives the values $r_{j}(x)=r_{11}$ on $\Gamma_{i j}$, the slave side.

Using the above scheme, we find $r_{11}$ on the boundary of each substructure $\Omega_{i}$. To get $r_{11} \in V_{11}$, we extend $r_{11}$, given on $\partial \Omega_{i}$, to $\Omega_{i}$ as a discrete harmonic function. For that, we solve $N$ independent problems of the form (7).

Summarizing, we see that the algorithm presented is very well suited for parallel computations. The number of iterations to get the solution with an accuracy $\varepsilon$ is independent of the jumps of $\rho(x)$ and depends only logarithmically on the ratio of $H$ and $h_{i}$.

\section{TECHNICAL TOOLS}

In this section a number of auxiliary results needed to prove Theorem 1 are formulated and proved. Let us first introduce certain operators similar to $L_{2}$ - projections. Let $p_{j}$ denote a projection from $L_{2}\left(\Gamma_{i j}\right)$ onto $M_{i j}^{h_{j}}\left(\Gamma_{i j}\right)$ given by

$\int_{\Gamma_{i j}} p_{j} v \Psi d s=\int_{\Gamma_{i j}} v \Psi d s, \quad \Psi \in M_{i j}^{h_{j}}\left(\Gamma_{i j}\right)$

It is easy to see that for $v \in H^{1}\left(\Gamma_{i j}\right)$

$\left\|v-p_{j} v\right\|_{L_{2\left(\Gamma_{i j}\right)}^{2}}^{2} \leq C h_{j}^{2}|v|_{H^{1}\left(\Gamma_{i j}\right)}^{2}$

and

$\left|p_{j} v\right|_{H^{1}\left(\Gamma_{i j}\right)}^{2} \leq C|v|_{H^{1}\left(\Gamma_{i j}\right)}^{2}$

To prove (26), we choose $v_{h} \in M_{i j}^{h_{j}}$ equal to $v$ at the interior nodal points of the $h_{j}$ triangulation of $\Gamma_{i j}$ and use the fact that $\left\|v-p_{j} v\right\|_{L_{2}} \leq\left\|v-v_{h}\right\|_{L_{2}}$. Using a standard interpolation theorem, element by element, we get (26). The estimate (27) follows from an inverse inequality and (26).

Let $\Pi_{j}$ be the projection from $L_{2}\left(\Gamma_{i j}\right)$ onto $W_{i j}^{h_{j}}\left(\Gamma_{i j}\right) \cap H_{0}^{1}\left(\Gamma_{i j}\right)$ defined by

$\int_{\Gamma_{i j}} \Pi_{j} v \Psi d s=\int_{\Gamma_{i j}} v \Psi d s, \quad \Psi \in M_{i j}^{h_{j}}\left(\Gamma_{i j}\right)$

Let $\Gamma_{i j}$, a side common to $\Omega_{i}$ and $\Omega_{j}$, be the master and slave side for $u_{i, h}$ and $u_{j, h}$ defined on $\Omega_{i}$ and $\Omega_{j}$, respectively. Let these functions satisfy the mortar condition:

$\int_{\Gamma_{i j}} u_{i, h} \Psi d s=\int_{\Gamma_{i j}} u_{j, h} \Psi d s, \quad \Psi \in M_{i j}^{h_{j}}\left(\Gamma_{i j}\right)$ 
Let $Q_{j}$ be the $L_{2}-$ projection from $H_{0}^{1}\left(\Gamma_{i j}\right)$ onto $\stackrel{0}{W_{i j}^{h_{i}}}\left(\Gamma_{i j}\right)$, i.e.

$\int_{\Gamma_{i j}} Q_{j} v \varphi d s=\int_{\Gamma_{i j}} v \varphi d s, \quad \varphi \in \stackrel{0}{W_{i j}^{h_{i}}}\left(\Gamma_{i j}\right)$

where $\stackrel{0}{W}_{i j}^{h_{i}}\left(\Gamma_{i j}\right)$ is a space of functions from $W_{i j}^{h_{i}}\left(\Gamma_{i j}\right)$ which vanish at the ends of $\Gamma_{i j}$.

In the lemmas below, we assume that $h_{i}$ and $h_{j}$ are of the same order; the constant $C$ is independent of $h_{i}$ and $h_{j}$.

Lemma 1 Let $u_{i, h}$ and $u_{j, h}$ vanish at the ends of $\Gamma_{i j}$ and satisfy (29), and let $\Gamma_{i j}$ be the master and slave sides of $\Omega_{i}$ and $\Omega_{j}$, respectively. Then

$\left\|u_{j, h}\right\|_{H_{00}^{1 / 2}\left(\Gamma_{i j}\right)}^{2} \leq C\left\|u_{i, h}\right\|_{H_{00}^{1 / 2}\left(\Gamma_{i j}\right)}^{2}$.

Remark 1 Lemma 1 follows from Lemma 1 of Belgacem (1995). Here we give an alternative proof, which is simpler.

Proof. We need to show only that

$\left\|\Pi_{j} u_{i, h}\right\|_{H_{00}^{1 / 2}\left(\Gamma_{i j}\right)}^{2} \leq C\left\|u_{i, h}\right\|_{H_{00}^{1 / 2}\left(\Gamma_{i j}\right)}^{2}$

since $\Pi_{j} u_{i, h}=u_{j, h}$ in view of $(28)$ and (29). We first note that

$\left\|\Pi_{j} u_{i, h}\right\|_{L_{2}\left(\Gamma_{i j}\right)}^{2} \leq 3\left\|u_{i, h}\right\|_{L_{2}\left(\Gamma_{i j}\right)}^{2}$.

This follows from (28) taking $\Psi$, here denoted by $v$, which is equal to $\Pi_{j} u_{i, h}$ at the nodal points of $\Gamma_{i j}$ and the fact that

$\left\|\Pi_{j} u_{i, h}\right\|_{L_{2}\left(\Gamma_{i j}\right)}^{2} \leq\left(\Pi_{j} u_{i, h}, v\right)_{L_{2}\left(\Gamma_{i j}\right)}=\left(u_{i, h}, v\right)_{L_{2}\left(\Gamma_{i j}\right)} \quad$ and

$\|v\|_{L_{2}\left(\Gamma_{i j}\right)}^{2} \leq 3\left\|\Pi_{j} u_{i, h}\right\|_{L^{2}\left(\Gamma_{i j}\right)}^{2}$

which are shown straightforwardly. For that, we use the fact that

$$
\begin{aligned}
\left(\Pi_{j} u_{i, h}, v\right)_{L^{2}\left(\Gamma_{i j}\right)=} & \int_{x_{0}+h_{j}}^{x_{N_{i j}}-h_{j}}\left(\Pi_{j} u_{i, h}\right)^{2} d s+ \\
& \frac{h_{j}}{2}\left\{\left(\Pi_{j} u_{i, h}\left(x_{0}+h_{j}\right)\right)^{2}+\left(\Pi_{j} u_{i, h}\left(x_{N_{i j}}-h_{j}\right)\right)^{2}\right\},
\end{aligned}
$$

where $x_{0}$ and $x_{N_{i j}}$ are the ends of $\Gamma_{i j}$.

Note that (32) is valid also for a $u_{i, h}$ which does not vanish at the ends of $\Gamma_{i j}$. That fact will be used below. 
Using the inverse inequality, we get

$$
\left\|\Pi_{j} u_{i, h}\right\|_{H_{00}^{1 / 2}\left(\Gamma_{i j}\right)}^{2} \leq C\left\{\frac{1}{h_{j}}\left\|\Pi_{j} u_{i, h}-Q_{j} u_{i, h}\right\|_{L_{2}}^{2}+\left\|Q_{j} u_{i, h}\right\|_{H_{00}^{1 / 2}}^{2}\right\} .
$$

Note that $\Pi_{j} Q_{j} u_{i, h}=Q_{j} u_{i, h}$. Using now the properties of $Q_{j}$, see e.g. Dryja and Widlund (1994), and (32), we get (31).

Let $\varphi_{i m}^{(s)}$ and $\varphi_{i m}^{(m s)}$ be the functions defined on $\Gamma_{i m}$, the slave side, and let $\varphi_{i l m}$ be defined on $\partial \Omega_{i}$; they have been introduced in Section 3 .

\section{Lemma 2}

$\left\|\varphi_{i m}^{(s)}\right\|_{L_{2}\left(\Gamma_{i m}\right)}^{2} \leq C h_{i}$.

Remark 2 This and the two next lemmas are proved in Achdou, Maday, and Widlund (1995). Here we give alternative proofs, which are simpler.

Proof. Let $\varphi_{i m}^{(s)}=z_{0}+z_{1}$ where $z_{0}$ is equal to $\varphi_{i m}^{(s)}$ at the ends of $\Gamma_{i m}$ and zero at the remaining nodal points. By the definition of $\varphi_{i m}^{(s)}$, see (11),

$\int_{\Gamma_{i m}} z_{0} \Psi d s+\int_{\Gamma_{i m}} z_{1} \Psi d s=0, \quad \Psi \in M_{i m}^{h_{i}}$

Let $\Psi=\tilde{z}_{1}$ be defined by the nodal values of $z_{1}$. ¿From (38) and using the $\varepsilon$-inequality, we get

$\int_{\Gamma_{i m}} z_{1} \tilde{z}_{1} d x=-\int_{\Gamma_{i m}} z_{0} \tilde{z}_{1} d x \leq \frac{1}{2 \varepsilon}\left\|z_{0}\right\|_{L_{2}}^{2}+\frac{\varepsilon}{2}\left\|\tilde{z}_{1}\right\|_{L_{2}}^{2}$.

Straightforwardly, using (35) for $z_{1}$ and $\tilde{z}_{1}$, we show that, cf. (34) and (33),

$\left\|\tilde{z}_{1}\right\|_{L^{2}\left(\Gamma_{i j}\right)}^{2} \leq 2 \int_{\Gamma_{i j}} z_{1} \tilde{z}_{1} d x$

Hence,

$\int_{\Gamma_{i m}} z_{1} \tilde{z}_{1} d x \leq C\left\|z_{0}\right\|_{L_{2}}^{2}$.

We also show straightforwardly, using again (35) for $z_{1}$ and $\tilde{z}_{1}$, that, cf. (33),

$\left\|z_{1}\right\|_{L_{2}\left(\Gamma_{i m}\right)}^{2} \leq\left(z_{1}, \tilde{z}_{1}\right)_{L_{2}\left(\Gamma_{i m}\right)}$.

Note that $\left\|z_{0}\right\|_{L^{2}}^{2} \leq C h_{i}$. Using these estimates, we get $\left\|\varphi_{i m}^{(s)}\right\|_{L_{2}}^{2} \leq\left\|z_{0}\right\|_{L_{2}}^{2}+\left\|z_{1}\right\|_{L_{2}}^{2} \leq C h_{i}$, which proves (37). 


\section{Corollary 1}

$\left\|\varphi_{i m}^{(s)}\right\|_{L^{\infty}\left(\Gamma_{i m}\right)}^{2} \leq \frac{C}{h_{i}}\left\|\varphi_{i m}^{(s)}\right\|_{L_{2}\left(\Gamma_{i m}\right)}^{2} \leq C$.

\section{Lemma 3}

$\left\|\varphi_{i m}^{(m s)}\right\|_{H_{00}^{1 / 2}\left(\Gamma_{i m}\right)}^{2} \leq C\left(1+\log \frac{H}{h_{i}}\right)$

Proof. Note that $\varphi_{i m}^{(m s)}=\Pi_{m} \varphi_{i m}^{(m)}$, where $\Pi_{m}$ is defined by (28).

Let $\varphi_{A}(x)$ be the nodal basis function defined on the triangulation of the master side, associated with the vertex $A=x_{i m l}$. We have,

$\left\|\Pi_{m} \varphi_{i m}^{(m)}\right\|_{H_{00}^{1 / 2}\left(\Gamma_{i j}\right)} \leq\left\|\Pi_{j}\left(\varphi_{i m}^{(m)}-\varphi_{A}\right)\right\|_{H_{00}^{1 / 2}\left(\Gamma_{i j}\right)}+\left\|\Pi_{j} \varphi_{A}\right\|_{H_{00}^{1 / 2}\left(\Gamma_{i j}\right)}$.

Using the inverse inequality and (32), valid also for functions which do not vanish at the ends of $\Gamma_{i m}$, we get

$\left\|\Pi_{m} \varphi_{A}\right\|_{H_{00}^{1 / 2}\left(\Gamma_{i j}\right)}^{2} \leq \frac{C}{h_{m}}\left\|\varphi_{A}\right\|_{L_{2}\left(\Gamma_{i j}\right)}^{2} \leq C$,

provided that $h_{i}$ and $h_{m}$ are of the same order. Using now (31), we get

$\left\|\Pi_{m}\left(\varphi_{i m}^{(m)}-\varphi_{A}\right)\right\|_{H_{00}^{1 / 2}\left(\Gamma_{i j}\right)}^{2} \leq C\left\|\varphi_{i m}^{(m)}-\varphi_{A}\right\|_{H_{00}^{1 / 2}\left(\Gamma_{i j}\right)}^{2}$.

The seminorm of $H^{1 / 2}$ is estimated by $C$ while the $L_{2}$-norm by $C\left(1+\log \frac{H}{h_{i}}\right)$; this follows from straightforward computations. Hence,

$\left\|\Pi_{m}\left(\varphi_{i m}^{(m)}-\varphi_{A}\right)\right\|_{H_{00}^{1 / 2}\left(\Gamma_{i j}\right)}^{2} \leq C\left(1+\log \frac{H}{h_{i}}\right)$.

Substituting this and (43) into (42), we get (41).

\section{Lemma 4}

$\left|\varphi_{i l m}\right|_{H^{1 / 2}\left(\partial \Omega_{i}\right)}^{2} \leq C\left(1+\log \frac{H}{h_{i}}\right)$.

Proof. Here there are three possibilities of the form $\varphi_{i l m}$ on $\partial \Omega_{i}$. If $\Gamma_{i m}$ and $\Gamma_{i l}$ are master sides of $\partial \Omega_{i}$, the estimate is obvious and without the logarithmic factor.

When the sides $\Gamma_{i l}$ and $\Gamma_{i m}$ are both slaves, the inequality follows from the inverse inequality, and Lemma 2 and 3.

Let $\Gamma_{i m}$ be a master side while $\Gamma_{i l}$ is a slave side. Let $I_{H} \varphi_{i l m}$ be the linear function with the same values as $\varphi_{i l m}$ at the vertices of $\Omega_{i}$. We have $\left|\varphi_{i l m}\right|_{H^{1 / 2}\left(\partial \Omega_{i}\right)}^{2} \leq 2 \| \varphi_{i l m}-$ $I_{H} \varphi_{i l m} \|_{H_{00}^{1 / 2}\left(\Gamma_{i l}\right)}^{2}+C$.

Note that $\varphi_{i l m}=\varphi_{i l}^{(s)}+\varphi_{l i}^{(m s)}$ on $\Gamma_{i l}$. The term with $\varphi_{i l}^{(s)}-I_{H} \varphi_{i l}^{(s)}$ is estimated straightforwardly by $C\left(1+\log \frac{H}{h_{i}}\right)$ using the inverse inequality and (40) while the term with $\varphi_{l i}^{(m s)}$ is handled by Lemma 3. Thus, $\left|\varphi_{i l m}\right|_{H^{1 / 2}\left(\partial \Omega_{i}\right)}^{2} \leq C\left(1+\log \frac{H}{h_{i}}\right)$ which shows (44). 
Lemma 5 For any $u_{i, h} \in W_{i j}^{h_{i}}\left(\Gamma_{i j}\right)$

$\left\|u_{i, h}\right\|_{L^{\infty}\left(\Gamma_{i j}\right)}^{2} \leq C\left(1+\log \frac{H}{h_{i}}\right)\left\|u_{i, h}\right\|_{H^{1 / 2}\left(\Gamma_{i j}\right)}^{2}$.

A proof of this fact is given in Dryja (1987)

\section{PROOF OF THEOREM 1}

For the proof of Theorem 1, we use the lemmas of Section 4. According to the general ASM theory, see Dryja, Smith, and Widlund (1994), it reduces to checking three key assumptions.

\section{Assumption (iii)}

It is obvious in our case since the space $V_{1}^{h}$ is decomposed into two only subspaces and $C_{0}^{2}=1$.

\section{Assumption (ii)}

We need to show that for $k=0,1$,

$a\left(u_{h}, u_{h}\right) \leq \omega b_{1 k}\left(u_{h}, u_{h}\right), \quad u_{h} \in V_{1 k}$.

For $k=1$, this follows from Lemma 1 and well known results on the matrix $K_{i j}^{1 / 2}$. Indeed, using an extension theorem, we have for $u_{h} \in V_{11}(\Omega)$

$\rho_{i}\left|u_{i, h}\right|_{H^{1}\left(\Omega_{i}\right)}^{2} \leq C \sum_{\Gamma_{i j} \subset \partial \Omega_{i}} \rho_{i}\left\|u_{i, h}\right\|_{H_{00}^{1 / 2}\left(\Gamma_{i j}\right)}^{2}$

Let $\Gamma_{i j}$ be the master side. Then,

$\rho_{i}\left\|u_{i, h}\right\|_{H_{00}^{1 / 2}\left(\Gamma_{i j}\right)}^{2} \leq C \rho_{i}\left(K_{i j}^{1 / 2} \underline{u}_{i, h}, \underline{u}_{i, h}\right)_{R^{N_{i j}}}$

see Dryja (1982). Let $\Gamma_{i j}$ be a slave side of $\Omega_{i}$, a side common with $\Omega_{j}$. Using Lemma 1 , we get

$\rho_{i}\left\|u_{i, h}\right\|_{H_{00}^{1 / 2}\left(\Gamma_{i j}\right)}^{2} \leq C \rho_{i}\left\|u_{j, h}\right\|_{H_{00}^{1 / 2}\left(\Gamma_{i j}\right)}^{2} \leq C \rho_{i}\left(K_{i j}^{1 / 2} \underline{u}_{j, h}, \underline{u}_{j, k}\right)_{R^{N_{i j}}}$

since $u_{i, h}$ and $u_{h, j}$ satisfy the mortar condition (2). By the selection of the master side, we have $\rho_{i} \leq \rho_{j}$. Hence,

$\rho_{i}\left\|u_{i, h}\right\|_{H_{00}^{1 / 2}\left(\Gamma_{i j}\right)}^{2} \leq C \rho_{j}\left(K_{i j}^{1 / 2} \underline{u}_{j, h}, \underline{u}_{j, h}\right)_{R^{N_{j i}}}$,

which proves (45) for $k=1$ with $\omega=$ constant. 
To show (45) for $k=0$, we first note that

$\left|u_{i, h}\right|_{H^{1}\left(\Omega_{i}\right)}^{2} \leq C\left|u_{i, h}-\bar{u}_{i, h}\right|_{H^{1 / 2}\left(\partial \Omega_{i}\right)}^{2}$

Any function $u_{h} \in V_{10}$ on $\partial \Omega_{i}$ can be represented as follows. Let $x_{i l m}, x_{i l p}, x_{i p m}$ be vertices of $\Omega_{i}$, denoted also by $A, B, C$, and let $\Gamma_{i m}, \Gamma_{i l}$, and $\Gamma_{i p}$ be the sides of $\Omega_{i}$ common with $\Omega_{m}, \Omega_{l}$, and $\Omega_{p}$. Using the functions introduced in Section 3, we see that $u_{i, h} \in V_{10}$ on $\partial \Omega_{i}$ is of the form

$u_{i, h}(x)=u_{i, h}(A) \varphi_{i l m}(x)+u_{i, h}(B) \varphi_{i l p}(x)+u_{i, h}(C) \varphi_{i p m}(x)$.

Note that $u_{i, h}$ on $\Gamma_{i l}$, the master side, is of the form

$u_{i, h}(x)=u_{i, h}(A) \varphi_{i l, A}^{(m)}+u_{i, h}(B) \varphi_{i l, B}^{(m)}$

while on $\Gamma_{i m}$, the slave side

$u_{i, h}(x)=u_{i, h}(A) \varphi_{i m, A}^{(s)}+u_{i, h}(C) \varphi_{i m, C}^{(s)}+u_{m, h}(A) \varphi_{i m, A}^{(m s)}(x)+u_{m, h}(C) \varphi_{i m, C}^{(m s)}(x)$

where $\varphi_{i l, A}^{(m)}, \varphi_{i m, A}^{(s)}$, and $\varphi_{i m, A}^{(m s)}$, are $\varphi_{i l}^{(m)}, \varphi_{i m}^{(s)}$ and $\varphi_{i m}^{(m s)}$ associated with the vertex $A$ etc. Here we consider the configuration where only $\Gamma_{i l}$ is a master side. A similar representation exists for $u_{i, h}$ for other configurations of master and slave sides of $\Omega_{i}$ and when $\Omega_{i}$ is a rectangle.

Note that the sum of the basis functions which appear in (51) is one. Using this and (52) and (53) in (50), and applying Lemmas 1 to 4, we get

$$
\begin{aligned}
\rho_{i}\left|u_{i, h}-\bar{u}_{i, h}\right|_{H^{1 / 2}\left(\partial \Omega_{i}\right)}^{2} \leq & C\left\{\sum_{x \in \nu_{i}}\left(1+\log \frac{H}{h_{i}}\right) \rho_{i}\left(u_{i, h}(x)-\bar{u}_{i, h}\right)^{2}\right. \\
& +\sum_{x \in \nu_{i m}}\left(1+\log \frac{H}{h_{m}}\right) \rho_{m}\left(u_{m, h}^{(m)}(x)-\bar{u}_{i, h}^{(s)}\right)^{2} \\
& \left.+\sum_{x \in \nu_{i p}}\left(1+\log \frac{H}{h_{p}}\right) \rho_{p}\left(u_{p, h}^{(m)}(x)-\bar{u}_{i, h}^{(s)}\right)\right\}
\end{aligned}
$$

where $\nu_{i m}$ and $\nu_{i p}$ are the ends of $\Gamma_{i m}$ and $\Gamma_{i p}$, the slave sides of $\Omega_{i}$ and $\bar{u}_{i, h}^{(s)}=\bar{u}_{i, h}$. Taking a sum in (54) with respect to $i$, we get the estimate (45) for $k=1$ with $\omega=$ constant. The proof of Assumption (ii) is complete.

\section{Assumption (i)}

We need to show that for any $u_{h} \in V_{1}^{h}$, there exists

$$
u_{h}=u_{h}^{(0)}+u_{h}^{(1)}, \quad u_{h}^{(0)} \in V_{10}, \quad u_{h}^{(1)} \in V_{11}
$$


such that

$\sum_{k=0}^{1} b_{k}\left(u_{h}^{(k)}, u_{h}^{(k)}\right) \leq C \delta^{2} a\left(u_{h}, u_{h}\right)$

Let $u_{h}^{(0)}$ be the interpolant of $u_{h} \in V_{1}^{h}$ belonging to $V_{10}$. Its representation on $\partial \Omega_{i}$ is given by (51) - (53). Let $u_{h}^{(1)}=u_{h}-u_{h}^{(0)} ;$ of course, $u_{h}^{(1)} \in V_{11}$. Let us first discuss $k=1$ and the form $b_{11}$ given by (13). It is known, see e.g. Dryja (1982), that

$\rho_{i}\left(K_{i j}^{1 / 2} \underline{u}_{i, h}^{(1)}, \underline{u}_{i, h}^{(1)}\right)_{R^{N_{i j}}} \leq C \rho_{i} \|\left. u_{i, h}^{(1)}\right|_{H_{00}^{1 / 2}\left(\Gamma_{i j}\right)} ^{2} \leq C\left(1+\log \frac{H}{h_{i}}\right)^{2} \rho_{i}\left|u_{i, h}\right|_{H^{1}\left(\Omega_{i}\right)}^{2}$

since $u_{h}^{(0)}$ on $\Gamma_{i j}$, as the master side, is a linear function. Summing this over the master sides, we get

$b_{11}\left(u_{h}, u_{h}\right) \leq C \delta^{2} a\left(u_{h}, u_{h}\right)$.

To check this estimate for the form $b_{11}$ given by (14) and (15), it is enough to see that

$b_{\Gamma_{i j}}\left(u_{i, h}^{(1)}, u_{i, h}^{(1)}\right) \leq C \rho_{i}\left\|u_{i, h}^{(1)}\right\|_{H_{00}^{1 / 2}\left(\Gamma_{i j}\right)}^{2}$.

We now show that, see (16),

$b_{10}\left(u_{h}^{(0)}, u_{h}^{(0)}\right) \leq C \delta^{2} a\left(u_{h}, u_{h}\right)$.

The first term of (16) is estimated by

$\sum_{x \in \nu_{i}} \rho_{i}\left(u_{i, h}(x)-\bar{u}_{i, h}\right) \leq C\left(1+\log \frac{H}{h_{i}}\right) \rho_{i}\left|u_{i, h}\right|_{H^{1 / 2}\left(\partial \Omega_{i}\right)}^{2}$

in view of Lemma 5 and Poincaré's inequality. To estimate the second term of (16), we again apply Lemma (5) and use the fact that the average values of $u_{i, h}^{(m)}$ and $u_{j, h}^{(s)}$ on $\Gamma_{i j}$ are equal to each other which follows from the mortar condition. Thus,

$\rho_{j}\left(u_{i, h}^{(m)}(x)-\bar{u}_{j, h}^{(s)}\right)^{2} \leq C\left\{\left(1+\log \frac{H}{h_{i}}\right) \rho_{i}\left|u_{i, h}\right|_{H^{1 / 2}\left(\partial \Omega_{i}\right)}^{2}+\left(1+\log \frac{H}{h_{j}}\right) \rho_{j}\left|u_{j, h}\right|_{H^{1 / 2}\left(\partial \Omega_{j}\right)}^{2}\right\}$.

Adding these two estimates and taking a sum with respect to $i$, we get (59). In turn, adding the estimates (58) and (59), we get (56). Assumption (i) have been checked. The proof of Theorem 1 is complete. 


\section{THE GEOMETRICALLY NONCONFORMING CASE}

In this section, we generalize the considerations from the previous sections to the case when the coarse partition is not triangulation. In the mortar element method this is called a geometrically nonconforming case. Unfortunately, it is not clear how to analyze ASM considered in Section 3 for the problem with the jump coefficients. Therefore here we discuss only elliptic problems with continuous coefficients. To simplify our presentation, we consider only the following problem:

Find $u \in H_{0}^{1}(\Omega)$ such that

$a(u, v)=f(v), \quad v \in H_{0}^{1}(\Omega)$,

$a(u, v)=\int_{\Omega} \nabla u \cdot \nabla v d x, \quad f(v)=\int_{\Omega} f v d x$.

The problem (60) is discretized by the mortar element method. For that let us, as in Section 2 , assume that $\Omega$ is polygonal region divided into subregions $\Omega_{i}$, which are triangles or rectangles. We make no assumption that this partition is a finite element triangulation. Let $H=\max _{i} H_{i}$ where $H_{i}$ is the diameter of $\Omega_{i}$. We now introduce a triangulation of each $\Omega_{i}$ with triangular elements and a parameter $h_{i}$. In general, this triangulation is nonmatching but we assume that the triangulation of each $\Omega_{i}$ is quasi-uniform, see Ciarlet (1978). Let $\Gamma_{i j}$ denoted the intersection of closures of $\Omega_{i}$ and $\Omega_{j}$; now this can be a part of sides of $\Omega_{i}$ and $\Omega_{j}$. The sides of $\Omega_{i}$ are denoted by $\gamma_{i j}$.

Let $X^{h}(\Omega)$ and $W_{i j}^{h_{i}}\left(\gamma_{i j}\right)$ be defined as in Section 2. Let us choose the master and slave sides $\gamma_{i j}$ of $\Omega_{i}$. Here the rule is that the mortar sides are entire sides of substructures, that they are disjoint, and that their union is equal to $\Gamma=\cup \partial \Omega_{i} \backslash \partial \Omega$. From this follows that the $\gamma_{i j}$, across the interface, is a union of $\Gamma_{i k}$, intersections of the closures of $\Omega_{k}$ and $\Omega_{i}$.

Let $\gamma_{i j}$ be the slave side of $\partial \Omega_{i}$ and let the test space, $M_{i j}^{h_{i}}\left(\gamma_{i j}\right)$, be defined as in Section 2 . We say that $u_{h} \in X^{h}$ satisfies the mortar condition on the slave side $\gamma_{i j}$ if

$\int_{\gamma_{i j}}\left(u_{i, h_{\mid \gamma_{i j}}}-\sum_{k} u_{k, h_{\mid \Gamma_{i k}}}\right) \Psi=0, \quad \Psi \in M_{i j}^{h_{i}}\left(\gamma_{i j}\right)$,

where the sum is taken over the $\Omega_{k}$ with nonzero intersection with $\gamma_{i j}$, i.e. over $\Gamma_{i k}$ such that $\gamma_{i j}=\cup \Gamma_{i k}$ (intersections with one point are excluded).

The space $V^{h}$ is defined as a space of functions from $X^{h}$ which satisfy condition (62) for each slave side $\gamma_{i j}$ belonging to interior of $\Omega$.

The discrete problem for (60) is of the form:

Find $u_{h} \in V^{h}$ such that

$a\left(u_{h}, v_{h}\right)=f\left(v_{h}\right)$

The problem has a unique solution and an estimate of the error is as in (4), see Bernardi, Maday and Patera (1990).

Our goal is to design and analyze an ASM like that in Section 3 for problem (63). The first question is that of the condition number of $A$, the matrix of the system resulting from (63). In Dryja and Widlund (1995), it has been established that also in the geometrically 
nonconforming case, the condition number of $A$ is as in (5) provided that the $h_{i}$ are of the order $h$.

Problem (63), as in Section 3, is reduced to a problem on the interfaces. Let $V_{1}^{h}$ be a space of functions belonging to $V^{h}$, which are discrete harmonic in each $\Omega_{i}$, cf. (8):

Find $u_{h} \in V_{1}^{h}$ such that

$s\left(u_{h}, v_{h}\right)=\tilde{f}\left(v_{h}\right), \quad v_{h} \in V_{1}^{h}$,

where $s\left(v_{h}, w_{h}\right)$ and $\tilde{f}\left(v_{h}\right)$ are the same as in Section 3 with the form $a(u, v)$ defined by $(61)$.

We now design ASM for solving (64) follows the scheme of Section 3.

The decomposition of $V_{1}^{h}$ is (cf. (9))

$V_{1}^{h}=V_{10}+V_{11}$.

Here $V_{11}$, as in Section 3 , is a space of functions from $V_{1}^{h}$ which vanish at vertices of $\Omega_{i}$ while $V_{10}$ is the generalization of the space from Section 3. It is defined as follows. Let $x_{i k l}$ be a vertex of $\Omega_{i}$ that is in common with the substructure $\Omega_{k}$ and others, that is an end point of $\gamma_{i j}$, a side of $\Omega_{i}$. The functions $\varphi_{i j}^{(s)}$ and $\varphi_{i j}^{(m)}$ are associated with $x_{i k l}$ and correspond to the slave and mortar sides $\gamma_{i j}$, respectively, are defined as in Section 3, i.e. $\varphi_{i j}^{(s)}$ is given by (11) while $\varphi_{i j}^{(m)}$ is linear with value one at $x_{i k l}$ and zero at the other end of $\gamma_{i j}$. The function $\varphi_{i j}^{(m s)}$ is defined by (10) where $\varphi_{i j}^{(m)}$ now is replaced by a sum of $\varphi_{i k}^{(m)}$ on the mortar sides of $\Omega_{k}$ which intersect $\gamma_{i j}$.

Using these functions, we define $\varphi_{i k l}$ which is associated with $x_{i k l}$ as follows. It is discrete harmonic in each $\Omega_{i}$ with data on $\partial \Omega_{i}$ which are zero except on the sides with the common vertex $x_{i k l}$. On these sides $\varphi_{i k l}$ is defined by: on the master side it equals $\varphi_{i k}^{(m)}$ while on the slave side it is equal to $\varphi_{i j}^{(s)}+\varphi_{i j}^{(m s)}$. Thus,

$V_{10}=\operatorname{span}\left\{\varphi_{i k l}\right\}$.

It is easy to see that $V_{10} \subset V_{1}^{h}$.

The bilinear forms $b_{1 k}, k=0,1$, defined over $V_{1 k} \times V_{1 k}$, are of the form:

For $\mathrm{k}=0$

$$
\begin{aligned}
b_{10}\left(u_{h}, u_{h}\right)= & \sum_{\Omega_{i}}\left\{\sum_{x \in \nu_{i}}\left(u_{i, h}(x)-\bar{u}_{i, h}\right)^{2}\right. \\
& \left.+\sum_{\gamma_{i j} \subset \partial \Omega_{i}}\left[\sum_{x \in \nu_{l i}}\left(u_{l, h}^{(m)}-\bar{u}_{i, h}^{(s)}\right)^{2}+\ldots+\sum_{x \in \nu_{p i}}\left(u_{p, h}^{(m)}-\bar{u}_{i, h}^{(s)}\right)^{2}\right]\right\} .
\end{aligned}
$$

Here $\Gamma_{l i}, \ldots, \Gamma_{p i}$ are parts of mortar sides given as intersections of $\Omega_{l}, \ldots, \Omega_{p}$ with $\gamma_{i j}$, the slave side of $\Omega_{i}$ and, as in Section 3, the $\nu_{l i}$ are the end points of the mortar side of $\Omega_{l}$ which contains $\Gamma_{l i}$ etc.

The form $b_{11}$ is similar to the one given by (13), i.e.

$$
b_{11}\left(u_{h}, v_{h}\right)=\sum_{\Omega_{i}} \sum_{\gamma_{i j} \subset \partial \Omega_{i}}\left(K_{i j}^{1 / 2} \underline{u}_{i, h}^{(m)}, \underline{v}_{i, h}^{(m)}\right)_{R^{N_{i j}}}
$$

where the sum is taken over the master sides $\gamma_{i j}$ of $\Omega_{i}$. 
Remark 3 The form $b_{11}\left(u_{h}, v_{h}\right)$ can be also defined by (14) where $b_{\Gamma_{i j}}$ is replaced by $b_{\gamma_{i j}}$.

Let

$T_{1}=T_{10}+T_{11}$

where the $T_{1 k}, k=0,1$, are defined by (18) with the forms $b_{1 k}$ introduced above.

Theorem 2 For any $v_{h} \in V_{1}^{h}$

$\delta^{-2} c a\left(v_{h}, v_{h}\right) \leq a\left(T_{1} v_{h}, v_{h}\right) \leq C \delta a\left(v_{h}, v_{h}\right)$

where $c$ and $C$ are positive constants independent of $H$ and $h_{i}, i=1, \ldots, N$, and $\delta=$ $\left(1+\log \frac{H}{h}\right)$ and $h=\inf _{i} h_{i}$.

The method can be implemented as described in Section 3.

\section{PROOF OF THEOREM 2}

We first formulate auxiliary results, which are needed to prove Theorem 2 .

The following lemma is the generalization of Lemma 1 to the geometrically nonconforming case.

Lemma 6 Let $u_{i, h}$ and $u_{l, h}, \ldots, u_{p, h}$ vanish at the vertices of $\Omega_{i}, \Omega_{l}, \ldots, \Omega_{p}$. Let $\gamma_{i j}$ be a slave side of $\Omega_{i}$ while $\gamma_{l j}, \ldots, \gamma_{p j}$ are master sides of $\Omega_{l}, \ldots, \Omega_{p}$ which intersect $\gamma_{i j}$. Under the condition (62), assuming that $h_{i}, h_{l}, \ldots, h_{p}$ are of the same order, it holds

$$
\begin{aligned}
\left\|u_{i, h}\right\|_{H_{00}^{1 / 2}\left(\gamma_{i j}\right)}^{2} \leq & C\left\{\left(1+\log \frac{H}{h_{l}}\right)\left\|u_{l, h}\right\|_{H_{00}^{1 / 2}\left(\gamma_{l j}\right)}^{2}+\ldots+\right. \\
& \left.+\left(1+\log \frac{H}{h_{p}}\right)\left\|u_{p, h}\right\|_{H_{00}^{1 / 2}\left(\gamma_{p j}\right)}^{2}\right\} .
\end{aligned}
$$

Proof. To simplify our presentation, let $\gamma_{i j}$ be a sum of only two $\Gamma_{k i}$ with $k=l$ and $k=p$, i.e. $\gamma_{i j}=\Gamma_{l i} \cup \Gamma_{p i}$ where $\Gamma_{l i} \subset \gamma_{l j}$ and $\Gamma_{p i} \subset \gamma_{p j}$. Let $u_{l p}$ be equal to $u_{l, h}$ on $\gamma_{l i}$ and be equal to $u_{p, h}$ on $\Gamma_{p i}$. Note that these functions do not vanish at the ends of $\gamma_{i j}$. Using the projection $\Pi_{i}$ introduced in Section 4 , see (28), we see that $u_{i, h}=\Pi_{i} u_{l p}$ on $\gamma_{i j}$ since $u_{i, h}$ and $u_{l, p}$ satisfy the mortar condition (62). We now show that

$$
\left\|\Pi_{i} u_{l p}\right\|_{H_{00}^{1 / 2}\left(\gamma_{i j}\right)}^{2} \leq C\left\{\left(1+\log \frac{H}{h_{l}}\right)\left\|u_{l, h}\right\|_{H_{00}^{1 / 2}\left(\gamma_{l j}\right)}^{2}+\left(1+\log \frac{H}{h_{p}}\right)\left\|u_{p, h}\right\|_{H_{00}^{1 / 2}\left(\gamma_{p j}\right)}^{2}\right\} .
$$

To see this, we introduce functions $z_{l}$ and $z_{p}$ defined as follows. Let the ends of $\gamma_{i j}$ be denoted by $A$ and $B$; they are in general not nodal points of the $h_{l^{-}}$and $h_{p}$-triangulations 
on $\gamma_{l j}$ and $\gamma_{p j}$. Let $A$ lie between $C$ and $D$, two neighboring nodal points of the $h_{l^{-}}$ triangulation. The function $z_{l}$ is equal to $u_{l, h}$ at $C$ and $D$ and zero at the remaining nodal points. In a similar way, we define $z_{p}$. Let $z_{l p}=z_{l}+z_{p}$. We have

$\left\|\Pi_{i} u_{l p}\right\|_{H_{00}^{1 / 2}\left(\gamma_{i j}\right)}^{2} \leq 2\left(\left\|\Pi_{i}\left(u_{l p}-z_{l p}\right)\right\|_{H_{00}^{1 / 2}}^{2}+\left\|\Pi_{i} z_{l p}\right\|_{H_{00}^{1 / 2}}^{2}\right)$.

Using the inverse inequality and the fact that $\Pi_{i}$ is $L_{2}$ - stable, see (32), we get

$\left\|\Pi_{i} z_{l p}\right\|_{H_{00}^{1 / 2}\left(\gamma_{i j}\right)}^{2} \leq \frac{C}{h_{i}}\left\|z_{l p}\right\|_{L_{2}\left(\gamma_{i j}\right)}^{2} \leq C\left(\left\|u_{l, h}\right\|_{L^{\infty}\left(\gamma_{l j}\right)}^{2}+\left\|u_{p, h}\right\|_{L^{\infty}\left(\gamma_{p j}\right)}^{2}\right)$.

provided that $h_{i}, h_{l}$, and $h_{p}$ are of the same order. Using Lemma 5 and the fact that $u_{l, h}$ and $u_{p, h}$ vanish at some points, we get

$\left\|\Pi_{i} z_{l p}\right\|_{H_{00}^{1 / 2}\left(\gamma_{i j}\right)}^{2} \leq C\left\{\left(1+\log \frac{H}{h_{l}}\right)\left|u_{l, h}\right|_{H^{1 / 2}\left(\gamma_{l j}\right)}^{2}+\left(1+\log \frac{H}{h_{p}}\right)\left|u_{p, h}\right|_{H^{1 / 2}\left(\gamma_{p j}\right)}^{2}\right\}$.

To estimate the first term of (73), we can use (31) since $u_{l p}-z_{l p}$ is a continuous function and vanishes at the ends of $\gamma_{i j}$. Thus,

$$
\begin{aligned}
\left\|\Pi_{i}\left(u_{l p}-z_{l p}\right)\right\|_{H_{00}^{1 / 2}\left(\gamma_{i j}\right)}^{2} & \leq C\left\|u_{l p}-z_{l p}\right\|_{H_{00}^{1 / 2}\left(\gamma_{i j}\right)}^{2} \leq \\
& \leq C\left(\left\|u_{l, h}-z_{l}\right\|_{H_{00}^{1 / 2}\left(\Gamma_{l i}\right)}^{2}+\left\|u_{p, h}-z_{p}\right\|_{H_{00}^{1 / 2}\left(\Gamma_{p i}\right)}^{2}\right) .
\end{aligned}
$$

It is easy to see that

$\left\|u_{l, h}-z_{l}\right\|_{H_{00}^{1 / 2}\left(\Gamma_{l i}\right)}^{2} \leq C\left(1+\log \frac{H}{h_{l}}\right)\left\|u_{l, h}\right\|_{H_{00}^{1 / 2}\left(\gamma_{l j}\right)}^{2}$,

since

$\left\|z_{l}\right\|_{H^{1 / 2}\left(\Gamma_{l i}\right)}^{2} \leq C\left\|u_{l, h}\right\|_{L^{\infty}\left(\gamma_{l j}\right)}^{2} \leq C\left(1+\log \frac{H}{h_{l}}\right)\left\|u_{l, h}\right\|_{H_{00}^{1 / 2}\left(\gamma_{l j}\right)}^{2}$

in view of Lemma 5 and Poincaré's' inequality. In a similar way, we estimate $u_{p, h}-z_{p}$. Substituting these estimates into (76), we get

$$
\begin{aligned}
\left\|\Pi_{i}\left(u_{l p}-z_{l p}\right)\right\|_{H_{00}^{1 / 2}\left(\gamma_{i j}\right)}^{2} \leq & C\left\{\left(1+\log \frac{H}{h_{l}}\right)\left\|u_{l, h}\right\|_{H_{00}^{1 / 2}\left(\gamma_{l j}\right)}^{2}+\right. \\
& \left.+\left(1+\log \frac{H}{h_{p}}\right)\left\|u_{p, h}\right\|_{H_{00}^{1 / 2}\left(\gamma_{p j}\right)}^{2}\right\} .
\end{aligned}
$$

In turn, substituting (75) and (77) into (73), we get (72).

Proofs of the two following lemmas are almost the same as the proofs of Lemmas 3 and 4 , respectively, therefore they are not given here. 


\section{Lemma 7}

$\left\|\varphi_{i l}^{(m s)}\right\|_{H_{00}^{1 / 2}\left(\gamma_{i j}\right)}^{2} \leq C\left(1+\log \frac{H}{h_{i}}\right)$.

\section{Lemma 8}

$\left|\varphi_{i l p}\right|_{H^{1 / 2}\left(\partial \Omega_{i}\right)}^{2} \leq C\left(1+\log \frac{H}{h_{i}}\right)$.

Proof of Theorem 2 We must check the three key assumptions as in Section 5.

Assumption (iii) obviously holds.

Assumption (ii). We need to show that for $k=0,1$, and $u_{h} \in V_{1 k}$

$a\left(u_{h}, u_{h}\right) \leq \omega b_{1 k}\left(u_{h}, u_{h}\right)$.

For $k=1$, it follows from Lemma 6 . Let $a_{i}\left(u_{h}, u_{h}\right)$ be the restriction of $a\left(u_{h}, v_{h}\right)$ to $\Omega_{i}$. We have

$a_{i}\left(u_{h}, u_{h}\right) \leq C \sum_{\gamma_{i j} \subset \partial \Omega_{i}}\left|u_{i, h}\right|_{H_{00}^{1 / 2}\left(\gamma_{i j}\right)}^{2}$.

For the mortar side, we use (49) while for the slave side we use Lemma 6. Substituting these estimates into (81) and summing the resulting inequality with respect to $i$, we get (80) with $\omega=C \delta$. To show (80) for $k=0$ and $u_{h} \in V_{10}$, we use a representation of $u_{h}$ on $\partial \Omega_{i}$ given by formulas similar to (51) - (53). Note that this time $u_{i, h}$, on the slave side $\gamma_{i j}$, is of the form

$$
\begin{aligned}
u_{i, h}(x) & =u_{i, h}(A) \varphi_{i j, A}^{(s)}+u_{i, h}(B) \varphi_{i j, B}^{(s)}+ \\
& +u_{l, h}(C) \varphi_{l i, C}^{(m s)}+u_{l, h}(D) \varphi_{l i, D}^{(m s)}+\ldots+u_{p, h}(E) \varphi_{p i, E}^{(m s)}+u_{p, h}(F) \varphi_{p i, F}^{(m s)},
\end{aligned}
$$

where $A$ and $B$ are the ends of $\gamma_{i j}$, the slave side of $\Omega_{i}$, while $C$ and $D$ are the ends of $\gamma_{l i}$, the mortar side of $\Omega_{l}$ which intersects $\Omega_{i}$, etc.

The function $u_{i, h}$ on the mortar side $\gamma_{i j}$ is linear, see (52).

Note also that the sum of basis functions used in the representation of $u_{i, h}$ on $\partial \Omega_{i}$ is equal to one. Using that and Lemmas 2, 6 to 8 , we show straightforwardly that

$$
\begin{aligned}
\left|u_{i, h}-\bar{u}_{i, h}\right|_{H^{1 / 2}\left(\partial \Omega_{i}\right)}^{2} \leq & C\left\{\left(1+\log \frac{H}{h_{i}}\right) \sum_{x \in \nu_{i}}\left(u_{i, h}-\bar{u}_{i, h}\right)^{2}+\right. \\
& +\sum_{\gamma_{i j} \subset \partial \Omega_{i}}\left[\sum_{x \in \nu_{l i}}\left(1+\log \frac{H}{h_{l}}\right)\left(u_{l, h}^{(m)}-\bar{u}_{l, h}^{(s)}\right)^{2}+\ldots+\right. \\
& \left.\left.+\sum_{x \in \nu_{p i}}\left(1+\log \frac{H}{h_{p}}\right)\left(u_{p, h}^{(m)}-\bar{u}_{i, h}^{(s)}\right)^{2}\right]\right\} .
\end{aligned}
$$


Summing this up with respect to $i$, we get (80) for $k=0$ with $\omega=C \delta$. The proof of Assumption (ii) is complete.

Assumption (i). We need to show (56) for the form $b_{1 k}\left(u_{h}, v_{h}\right)$ defined in Section 6, see (67) and (68). Let $u_{h}^{(0)} \in V_{10}$ be the interpolant of $u_{h} \in V_{1}^{h}$ and $u_{h}^{(1)}=u_{h}-u_{h}^{(0)}$. Of course, $u_{h}^{(1)} \in V_{11}$. The estimate

$b_{11}\left(u_{h}^{(1)}, u_{h}^{(1)}\right) \leq C \delta^{2} a\left(u_{h}, u_{h}\right)$

is proved as in Section 5, using Lemma 6.

To show that

$b_{10}\left(u_{h}^{(0)}, u_{h}^{(0)}\right) \leq C \delta a\left(u_{h}, u_{h}\right)$

we proceed in a way similar to that in Section 5. Adding (84) and (85), we get the estimate by checking Assumption (i). The proof of Theorem 2 is complete.

\section{REFERENCES}

Achdou, Y., Maday, Y., and Widlund, O. (1995) Substructuring preconditioners for the mortar method in two dimensions. Tech. Rep., Courant Institute (to appear).

Belgacem, F. (1995) The mortar finite element method with Lagrange multipliers. Methods in Applied Mechanics and Engineering (to appear).

Bernardi, Chr. and Maday, Y. (1995) Mesh adaptivity in finite elements by the mortar method. Tech. Rep. R 94029, Université Pierre et Marie Curie and CNRS.

Bernardi, Chr., Maday, Y., and Patera, A. (1994) A new nonconforming approach to domain decomposition: the mortar element method. Pitman, Brezis, H. and Lions J.L., eds.

Ciarlet, P.G. (1978) The finite element method for elliptic problems. North-Holland, Amsterdam.

Dryja, M. (1982) A capacitance matrix method for Dirichlet problem on polygonal region. Numer. Math. 39, 51-64.

Dryja, M. (1988) A method of domain decomposition for 3-D finite element problems. In First International Symposium on Domain Decomposition Methods for PDEs, Glowinski, A., Golub, G.H., Meurant, G.A. and Périaux, J., eds., SIAM, Philadelphia, PA, 43-61.

Dryja, M., Smith, B., and Widlund, O. (1994) Schwarz analysis of iterative substructuring algorithms for elliptic problems in three dimensions. SIAM J. Numer. Anal. 31, 1662-1694.

Dryja, M. and Widlund, O. (1995) A bound of the condition number of mortar finite element stiffness matrices (in preparation).

Widlund, O. (1995) Domain decomposition methods for spectral and mortar finite element approximation of elliptic problems. Proceedings of the Third International Congress on Industrial and Applied Mathematics, Hamburg, June 1995 (to appear). 